\title{
Damage spreading on two-dimensional trivalent structures with Glauber dynamics: Hierarchical and random lattices
}

\author{
Z. Z. Guo, ${ }^{*}$ K. Y. Szeto, ${ }^{\dagger}$ and Xiujun $\mathrm{Fu}^{\ddagger}$ \\ Department of Physics, Hong Kong University of Science and Technology, Clear Water Bay, Hong Kong, China
}

(Received 8 March 2004; published 2 July 2004)

\begin{abstract}
The damage spreading of the Ising model on various two-dimensional trivalent structures with Glauber dynamics is investigated. It is shown that topology plays an important role in determining the damage spreading transition temperatures of the trivalent structures. When damage is considered in terms of only the topological properties of the cellular patterns, the transition temperature above which damage is saturated is found to be determined by the cells with the highest edge number. When the area of cells is also taken into account in the computation of damage, the damage spreading transition temperatures are all lowered. These results are verified by simulation on a set of hierarchical lattices constructed by recursive application of the star-triangle transformation on the vertices of the hexagonal structure, as well as soap froth and randomized lattice structures using Voronoi construction.
\end{abstract}

DOI: $10.1103 /$ PhysRevE.70.016105

PACS number(s): $05.50 .+\mathrm{q}, 75.10 . \mathrm{Hk}, 75.40 . \mathrm{Mg}, 75.10 . \mathrm{Nr}$

\section{INTRODUCTION}

Damage spreading (DS) in the Ising model with a given dynamics has been studied extensively. DS is a useful technique in the description of the evolution of perturbation spreading through a cooperative system [1] or random growth process [2]. In terms of application, the significance of DS can be found in its relevance in the research in many economic and social phenomena. For example, it may be useful in business applications when one wants to estimate and thereby control the damage incurred by a sudden change of alliance of a particular site on the supermarket network or shopping mall from company Red to company Blue. The study of damage spreading on point patterns using different dynamics may help us to get a better theoretical understanding of the competitions between companies in a planar network of shops or centers.

Many elements which characterize the damage spreading process have been considered in the literature, including the interactions (ferromagnetic [3], antiferromagnetic [4], spin glass [5], etc.), the Monte Carlo rules (heat bath [6], Glauber [4], Metropolis [7], etc.), the lattice geometry (square [1,3], triangle [4], cubic [8], etc.), and the symmetry of the spin variables and the external conditions (e.g., magnetic field [9]). However, none of these works take the topology of the structures into consideration. In this paper we will study the DS on several trivalent structures, as they are topological stable.

In recent years, much attention has been paid to the trivalent cellular structures such as soap froth and Voronoi $[10,11]$, which tile the plane with irregular polygons which have different areas. These structures have the generic fea-

\footnotetext{
*Permanent address: Department of Physics, Inner Mongolia Normal University, Hohhot, P. R. China, 010022

${ }^{\dagger}$ Corresponding author.

${ }^{*}$ Permanent address: Department of Physics, South China University of Science and Technology, Guangzhou, China.
}

ture of random tiling, so that they may be more relevant for application in econophysics where random point patterns are of primary importance. Other kinds of interesting trivalent structures constructed by polygons with different areas is the hierarchical regular lattices, such as the 4-8 lattice $[12,13]$ and the 4-6 lattice [14], which tile the plane with regular polygons of two kinds. In this paper, we will present a new set of hierarchical regular lattices-the 3-12, the 3-6-24, and the 3-6-12-48 lattices which are generated from the hexagonal lattice by the star-triangle transformation. The DS of Ising model on both the hierarchical, soap, and Voronoi structures are studied with Glauber dynamics. We mainly address here the transition temperatures above which damage spreading will saturate in various topologies, with and without considering the distribution of areas of the cells in the trivalent patterns. In Sec. II, we discuss the hierarchical and the Voronoi structures. We then discuss the dynamics of damage spreading in an Ising model in Sec. III. The results are summarized in Sec. IV, where heuristic arguments are provided to explain the observed transition temperatures. Area influence on the interaction strength in the Ising model is discussed in Sec. V.

\section{HIERARCHICAL AND VORONOI STRUCTURES}

Since the hexagonal cellular structure is dual to the triangular lattice, we consider the construction of a set of hierarchical trivalent cellular structure based on the star-triangle transformation operating on the vertices of the hexagons. The simplest trivalent regular lattice is the pure hexagonal lattice [Fig. 1(a)]. On each vertex of the hexagon, we replace the star by a triangle, so that we have a triangle on each vertex of the original hexagon. (This star-triangle transformation is often used in decimation procedures.) [15] The result is a crystal made with 12-gons and triangles. Our choice on the edge length of the 12-gon is such that it is regular. Following the terminology of Liebmann [12] for the 4-8 lattice, we call this two-dimensional structure the 3-12 lattice [Fig. 1(b)]. By repeating this procedure on the triva- 


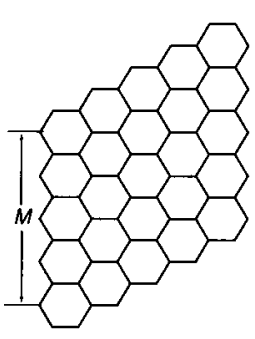

(a)

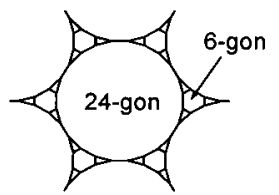

(d)

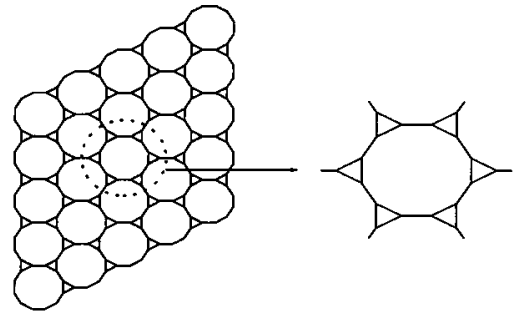

(b)

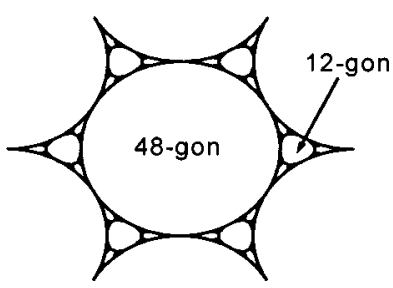

(e)
FIG. 1. Hierarchical trivalent structures generated by the startriangle transformation.(a) The pure hexagonal lattice, (b) the 3-12 lattice, generated by the first-order star-triangle transformation (c) the primary cell of the 3-12 lattice, (d) the primary cell of the 3-6-24 lattice and (e) the primary cell of the 3-6-12-48 lattice

lent vertex, we can get the 3-6-24 lattice composed with 24-gon, 6-gon, and triangles (after the second order startriangle transformation) and the 3-6-12-48 lattice composed with 48-gon, 12-gon, 6-gon, and triangles (after the third order star-triangle transformation), etc.

The number of triangles in these structures are, respectively, $0,2 M^{2}, 6 M^{2}$, and $18 M^{2}$, while the total numbers of cells in the corresponding lattices are $M^{2}, M^{2}+2 M^{2}, M^{2}$ $+2 M^{2}+6 M^{2}$, and $M^{2}+2 M^{2}+6 M^{2}+18 M^{2}$, where $M$ is the size of the lattice (Fig. 1). Thus, the numbers of triangles in hexagons, 3-12, 3-6-24, 3-6-12-48 lattices over the total number of cells are $0,2 / 3,2 / 3,2 / 3$. This means that the fraction of triangles in the 3-12, 3-6-24, 3-6-12-48 is a constant $2 / 3$. We will see whether the small cells like the triangles play any role in the spreading of damage in the next section. For the record, the probability distribution of edge number in these cells are given as follows. 3-12 lattice: $p(3)=2 / 3, p(12)=1 / 3$; 3-6-24 lattice: $p(3)=2 / 3, p(6)=2 / 9$, $p(12)=1 / 9$; and 3-6-12-48 lattice: $p(3)=2 / 3 ; p(6)=2 / 9$; $p(12)=2 / 27 ; p(48)=1 / 27$. The average coordination numbers in all these hierarchical structures are 6.

We also construct the Voronoi patterns from sets of points randomly distributed in the plane. Different control levels on the minimal distance between any two points are used to generate different Voronoi cellular patterns $[10,11]$. We sequentially place a point randomly on the unit square and calculate the minimum distance $d_{i}$ between this point $\mathrm{i}$ and all other existing points on the square. If $d_{i}>d_{c}$, which is a cutoff distance less than $1 / \sqrt{N}$, we accept this point, otherwise not. This process continues till we get $N$ points on the square. The soap structures are obtained by experiments which are described in our previous papers [10,11].The structure of soap is similar to that of Voronoi's.

\section{GLAUBER DYNAMICS FOR DAMAGE SPREADING}

We use the Monte Carlo method to investigate damage spreading in these two-dimensional trivalent structures. We put the spin $s=\{ \pm 1\}$ on the center of each cell of the pattern, forming an Ising model defined on the dual lattice. We consider the Hamiltonian of nearest-neighbor Ising model to be of the form

$$
H=-\sum_{\langle i, j\rangle} J_{i j} s_{i} s_{j},
$$

where $J_{i j}>0$ is the ferromagnetic exchange interaction coefficient between the nearest-neighbor sites $i$ and $j$, i.e., the interaction between the nearest-neighbor agents (polygons). It is natural to relate $J_{i j}$ with their areas. In order to compare the DS with and without considering the area influence, two cases are considered in the calculation: $J_{i j}=J$ and $J_{i j}$ $\propto\left(A_{i}, A_{j}\right)$, where $A_{i}$ is the area of the $i$ th cell. The first case is straightforward as there is no area influence. In the latter case, which includes the area influence, we illustrate the interaction with an example for the 3-12 lattice. The interaction between the large cells (both cells $i$ and $j$ are 12-gons), is taken as $J_{i j}=J$. For interaction between one small and one large cell (cell $i$ is a triangle and cell $j$ a 12-gon), we take $J_{i j}=J^{*} A_{3} / A_{12}$. In general, the interaction of cell $i$ and $j$ of different areas $\left(A_{j}>A_{i}\right)$ is reduced by the ratio of their area $J_{i j}=J\left(A_{i} / A_{j}\right)$. This consideration of area factor in the ferromagnetic coupling has an interesting interpretation in econophysics $[16,17]$. One can interpret the area of a cell as the customer base, or resource, of a particular agent in a multiagent system. The interaction between agents (or cells) therefore depends quite logically on their power, as measured by the resource or customer base. For more application of this model, please refer to Ref. [16].

For damage spreading, we first consider a system A which evolves for a long time to reach equilibrium. We then produce a replica $\mathrm{B}$ of the system $\mathrm{A}$ at equilibrium. At $t=0$, the spin in one of the cell (we here generically refer it as the center cell) in B is flipped (damaged) and fixed forever after. The Hamming distance (or damage) in phase space for these two trivalent structures as a function of time is calculated by

$$
D(t)=\frac{1}{N} \sum_{i=1}^{N}\left(1-\delta_{s_{i}^{A}(t), s_{i}^{B}(t)}\right),
$$

where $\left\{s_{i}^{A}(t)\right\}$ and $\left\{s_{i}^{B}(t)\right\}$ are the two spin configurations of the system which subject to the same thermal noise and the same set of random number and $N$ is the number of the total spins on the lattice studied. In this paper, we employ Glauber dynamics for the evolution of the spin configuration $s$ $=\left\{s_{i}(t)\right\}$, with the transition probability of flipping spin $i$ given by [18] 


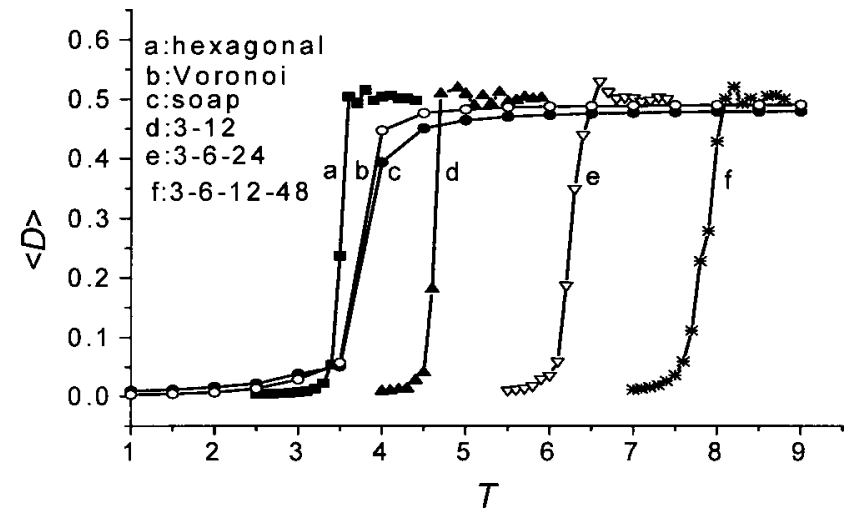

FIG. 2. Averaged damage spreading of various trivalent structures as the function of temperature. $T$ is in units of $J k_{B}, M=20,15$, 15 , and 15 for the hexagonal 3-12, 3-6-24, and 3-6-12-48 lattice. Details of the Voronoi and soap froth are given in the text.

$$
w_{i}(s)=\min \left[1, \exp \left(-\frac{\Delta E_{i}}{k_{B} T}\right)\right],
$$

where $\Delta E_{i}$ is the change in energy when spin $i$ is flipped. The results are averaged over 100 configurations.

\section{NUMERICAL RESULTS}

First let us consider the simple case $J_{i j}=J$. The results of the averaged damage spreading of various trivalent structures as the function of temperature are shown in Fig. 2. We can say that $T_{s}(6)<T_{s}(12)<T_{s}(24)<T_{s}(48)$, where $T_{s}(6)$, $T_{s}(12), T_{s}(24)$, and $T_{s}(48)$ stand for the DS transition temperatures corresponding to the pure hexagonal, 3-12, 3-6-24, and the 3-6-12-48 lattice. Thus, the more complicated the lattice is, the higher the transition temperature for damage spreading. We can understand this trend from two different points of view. The first one is to consider the triangles in the lattices. Note that from the zero order to the third order startriangle transformation, the number of the triangles in corresponding lattice are $0,2 M^{2}, 6 M^{2}$, and $18 M^{2}$, where $M$ is the size of the lattice [the number of the main polygons along one direction, see Fig. 1(a)]. Since we have neglected the area influence on the DS in the calculation, we give the triangle the same level of importance as other polygons. Now when the triangle is damaged, at most three neighboring cells will be affected, so that the spreading of damage is more difficult when other cells with higher edge number, such as the 6-gon, 12-gon, 24-gon, or the 48-gon are damaged. In other words, for the lattices with many triangles, higher temperatures are needed to reach the equilibrium state, resulting in the larger $T_{s}$.

From another point of view, the DS transition temperature, which is a measure of the thermal noise needed to spread damage, will be very much dependent on the condition of those cells with highest edge number. Once the cells with highest edge number (we call them the main cells) are damaged, the spreading is much faster as they have the most number of neighbors. Since our simple model does not take into account the area of the cell, the relative rarity of the

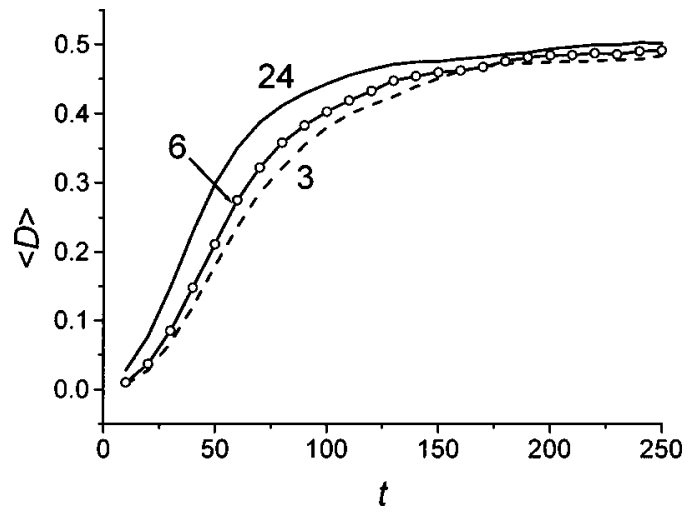

FIG. 3. Time dependence of the averaged damage spreading of the 3-6-24 lattice at $T=7$. "3," "6," and " 24 " indicates that a triangle, a hexagon, or a 24-gon in the center area of the lattice is damaged and fixed in configuration $\mathrm{B}$.

main cell (or equivalently the relative abundance of triangles) in our hierarchical structures make damage spreading more difficult in structures with main cells with higher edge number. Thus, in either viewpoint, we expect the critical temperature for damage spreading rise with the edge number of the main cell of a given lattice.

In our simulation, we have considered various choices of cells as the center cell. The results are similar so that in our presentation, the data represent those cases where the main cell is the center cell where the spin is fixed in a given state in configuration B. No obvious difference occurs if we fix polygons with smaller contiguity number $f$ in the center area of the lattices in the long time limit, because the memory of the original damaged cell should be lost after a sufficiently long time. But if we focus our attention on the beginning of the damage spreading, the time dependence of damage will show the dependence of damage on topology. Figure 3 shows the time dependence of damage at a given temperature for the cases we choose different cells as the central damaged cell in the 3-6-24 lattice. Intuitively, the more linkage a cell has, when it is damaged, the more cells will be affected more quickly. So when we use the 24-gon as the center cell for the fixed spin in configuration $\mathrm{B}$, the system reach the saturated damage value fastest.

Physically, damage measures the fractions of spins that are different in configurations $\mathrm{A}$ and $\mathrm{B}$. At higher temperature $\left(T>T_{s}\right)$ and in long time limit, we observe that the damage value saturates at $\langle D(t \rightarrow \infty)\rangle=0.5$ for all lattices [4]. Since the spins are put at the centers of the polygons, our pure hexagonal lattice is in fact of the same topology with the triangular lattice in which the spins are put on the vertices. Both of them have six neighbors. Our result for the hexagonal lattice yields a $T_{s}=3.6-3.7$, which compares well with the results of 3.64096 for the ferromagnetic Ising phase transition critical temperature in the triangular lattice according to Ref. [4].

Another important feature we can see from Fig. 2 is that the DS of the soap froth and Voronoi behave very similar to the pure hexagonal lattice. The reason is easy to understand. Figure 4 shows the distribution of polygons in Voronoi. Although the Voronoi is made with various polygons, the most 


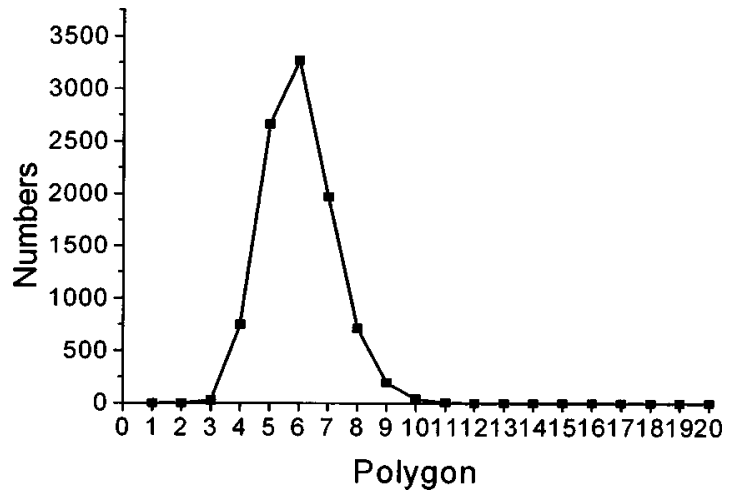

FIG. 4. Distribution of numbers of the polygons in a Voronoi pattern.

common polygons are hexagons. Similar property is found in soap froth. Thus, these three kinds of lattices have nearly the same topology. On the other hand, the number of polygons with $f<6$ in Voronoi and soap is not negligible, so the DS transition temperatures in Voronoi and soap are slightly bigger than that of the pure hexagonal lattice.

The Voronoi pattern used in Fig. 2 and 4 is generated under the condition $F=0.4$ [10]. Here $F=d_{c} \sqrt{N}$ is the factor with the minimal distance between any two points. The soap pattern used here is from the experimental data No.0901_09 [11]. Other Voronoi or soap structures are also calculated and their critical temperatures of the dynamical transition are approximately the same.

\section{AREA FACTOR IN DAMAGE}

Now we consider the area influence on the interaction coefficient between the nearest-neighbor sites (polygons).
Let us assume $J_{i j}=J\left[\min \left(A_{i}, A_{j}\right) / \max \left(A_{i}, A_{j}\right)\right]$. Similar phase transition phenomena for damage spreading are observed in various trivalent structures in the long time limit but the DS transition temperatures are obviously lowered, as shown in Fig. 5.

First, we compare the DS transition temperatures of the trivalent regular lattices. The DS transition temperatures of the 3-12 lattice for the cases $J_{i j}=J$ and $J_{i j}$ $=J\left[\min \left(A_{i}, A_{j}\right) / \max \left(A_{i}, A_{j}\right)\right]$ are $4.8 J$ and $3.4 J$, respectively. For the 3-6-24 lattice, these values are $6.25 \mathrm{~J}$ and $3.45 \mathrm{~J}$, respectively. We see that the DS transition temperatures in both lattices with area factors are very close, approximately 3.4J. Again, let us take the simple case of 3-12 lattice as example to explain this result. Since $A_{12}>>A_{3}$, the interaction between the 12-gon and the triangle is very small and can be neglected. The lattice is seen being made of "pure" 12-gons but the contiguity number of the 12-gons is only 6 due to the approximate breakdown of their interactions with triangles. So the DS behavior is very similar to the pure hexagonal lattice. The DS transition temperature approaches the pure hexagonal lattice case. The situations are the same for the 3-6-24 lattice and the 3-6-12-48 lattice.

As for the Voronoi and soap structures, we can see from Figs. 5(c) and 5(d) that the DS transition temperatures of the case $J_{i j}=J\left[\min \left(A_{i}, A_{j}\right) / \max \left(A_{i}, A_{j}\right)\right]\left(T_{s}=a J\right.$ with $a=2.1$ and 2.2 for Voronoi and soap, respectively) are lowered compared with the case $J_{i j}=J(a=3.7$ and 3.75 for Voronoi and soap, respectively). Since the areas of the polygons with larger contiguity number are bigger than that of the polygons with smaller contiguity number on average (Lewis law [19] ) , the role of the polygons with larger contiguity number is enhanced in the case $J_{i j}=J\left[\min \left(A_{i}, A_{j}\right) / \max \left(A_{i}, A_{j}\right)\right]$. The damage spreads faster due to the enhanced linkage of cells. That

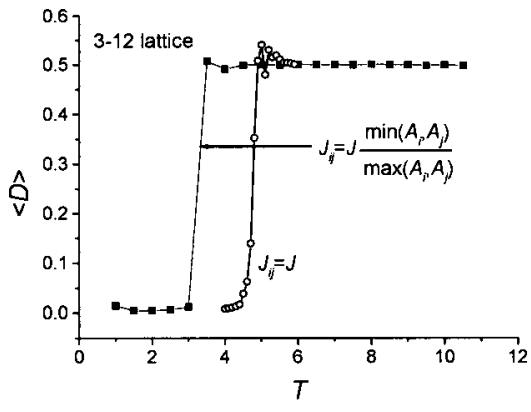

(a)

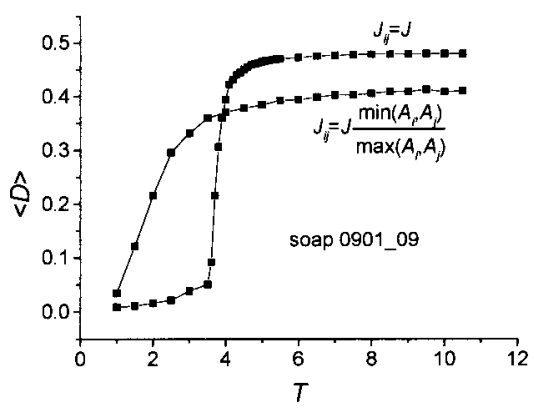

(c)

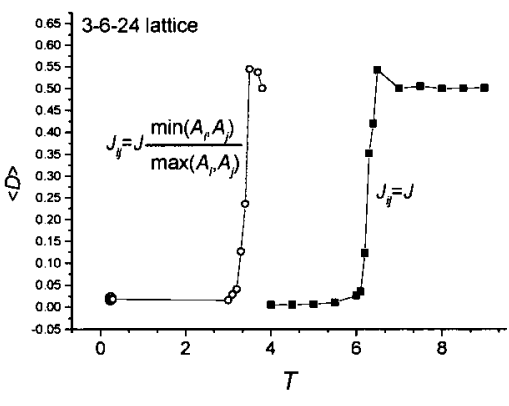

(b)

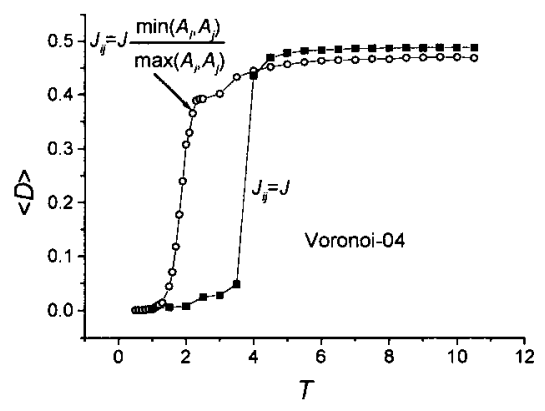

(d)
FIG. 5. Comparison of the phase transition curves of the various trivalent structures for the cases $J_{i j}=J$ and $J_{i j}$ $=J\left[\min \left(A_{i}, A_{j}\right) / \max \left(A_{i}, A_{j}\right)\right]$. 
is why the DS transition temperatures are lowered in the $J_{i j}=J\left[\min \left(A_{i}, A_{j}\right) / \max \left(A_{i}, A_{j}\right)\right]$ case.

\section{DISCUSSION}

In summary, we have studied the damage spreading of the Ising model on the two-dimensional trivalent structures with Glauber dynamics. We find that topology plays an important role in determining the damage spreading transition temperatures of the trivalent structures. If we neglect the influence of cell areas on damage spreading and only consider the topology, we observe that those patterns with more cells (polygons) with small edge number have larger transition tem- peratures, or equivalently, those patterns with main cell that has higher edge number will have higher transition temperatures. When the area influence is taken into consideration together with the edge number, the transition temperatures in general are all lowered. In this case, the damage spreading on the regular trivalent lattices are similar to that on the pure hexagonal lattice. A more detailed analysis, using MigdalKadanoff transformation, will be conducted to relate models with area dependent interaction to the hexagonal model.

\section{ACKNOWLEDGMENTS}

K.Y. Szeto acknowledges the support of RGC Grant No. HKUST 6071/02P.
[1] H. Eugene Stanley, Dietrich Stauffer, Janos Kertesz, and Hans J. Herrmann, Phys. Rev. Lett. 59, 2326 (1987).

[2] The Monte Carlo Method in Condensed Matter Physics, edited by K. Binder (Springer-Verlag, Berlin 1992), pp. 93-120.

[3] F. G. Brady Moreira, Adauto J. F. de Souza, and Ananias M. Mariz, Phys. Rev. E 53, 332 (1996).

[4] F. D. Nobre, A. M. Mariz, and E. S. Sousa, Phys. Rev. Lett. 69, 13 (1992).

[5] B. Derrido and G. Weisbuch, Europhys. Lett. 4, 657 (1987).

[6] A. Coniglio, L. de Arcangelis, H. J. Herrmann, and N. Jan, Europhys. Lett. 8, 315 (1989).

[7] Ge-Jun Liu, H.-B. Schutter, and Jia-Zhen Hu, Commun. Theor. Phys. 35, 480 (2001).

[8] Uriel M. S. Costa, J. Phys. A 20, L583 (1987).

[9] G. L. e Caer, J. Phys. A 22, L647 (1989).

[10] K. Y. Szeto, Xiujun Fu, and W. Y. Tam, Phys. Rev. Lett. 88, 138302 (2002).
[11] K. Y. Szeto and W. Y. Tam, Phys. Rev. E 53, 4213 (1996).

[12] R. Liebmann, Statistical Mechanics of Periodic Frustrated Ising systems, edited by $\mathrm{H}$. Araki, et al. in Lecture Notes in Physics, (Springer-Verlag, Berlin, 1986).

[13] K. Y. Lin and C. C. Chang, Int. J. Mod. Phys. B 16, 1241 (2002).

[14] J. Oitmaa and M. Keppert, J. Phys. A 35, L219 (2002).

[15] C. Itzykson and J-M. Drouffe, Statistical Field Theory (Cambridge University Press, Cambridge, 1989), Vol. 1, p. 184.

[16] K. Y. Szeto and Chiwah Kong, J. Comp. Econ 22(2). 163 (2003).

[17] Chiwah Kong, Master thesis, Department of Physics, Hong Kong University of Science and Technology, Hong Kong, 2002.

[18] B. C. S. Grandi and W. Figueiredo, Phys. Rev. E 56, 5240 (1997).

[19] D. Lewis, Anat. Rec. 38, 351 (1928). 